Relations industrielles

Industrial Relations

\title{
Soares, Angelo (sous la direction de), Stratégies de résistance et travail des fermmes
}

\section{Marianne Kempeneers}

Volume 54, numéro 2, 1999

URI : https://id.erudit.org/iderudit/051244ar

DOI : https://doi.org/10.7202/051244ar

Aller au sommaire du numéro

Éditeur(s)

Département des relations industrielles de l'Université Laval

ISSN

0034-379X (imprimé)

1703-8138 (numérique)

Découvrir la revue

Citer ce compte rendu

Kempeneers, M. (1999). Compte rendu de [Soares, Angelo (sous la direction de), Stratégies de résistance et travail des femmes]. Relations industrielles / Industrial Relations, 54(2), 420-421. https://doi.org/10.7202/051244ar

Tous droits réservés @ C Département des relations industrielles de l'Universite Laval, 1999
Ce document est protégé par la loi sur le droit d'auteur. L’utilisation des services d'Érudit (y compris la reproduction) est assujettie à sa politique d'utilisation que vous pouvez consulter en ligne.

https://apropos.erudit.org/fr/usagers/politique-dutilisation/ 


\section{Stratégies de résistance et travail des femmes}

publié sous la direction d'Angelo SOARES, Montréal-Paris : L'Harmattan, 1997, 304 p., ISBN 2-89489-019-2.

Les textes réunis dans ce collectif ont été présentés au $64^{\mathrm{e}}$ Congrès de l'ACFAS (Association canadienne française pour l'avancement des sciences) en mai 1996 , au colloque "Stratégies de résistance et travail des femmes". C'est à l'initiative d'Angelo Soares que nous devons l'organisation de ce colloque et l'élaboration de cet ouvrage.

Dans le contexte bien documenté de la persistance d'une division sexuelle du travail à l'échelle mondiale et des conséquences qui s'ensuivent pour les femmes (concentration dans les emplois moins avantageux, aux conditions plus précaires et moins rémunérés que les emplois occupés par les hommes), il s'agit ici d'appréhender le travail des femmes à travers les diverses stratégies que cellesci utilisent pour accéder et demeurer en emploi tout en contestant les rapports de pouvoir inhérents à l'organisation du travail. On ne parle pas ici de mouvements de résistance collectifs et organisés, comme les grèves par exemple, mais bien de formes individuelles et quotidiennes de lutte silencieuse, de résistance au travail. Les textes présentés ont en commun de mettre en évidence le rôle actif des travailleuses dans l'univers de travail et ainsi démasquer la fausse image de la passivité des femmes.

Comme le souligne Maria de Koninck dans la postface de l'ouvrage, on a là matière à réflexion concernant l'orientation des recherches féministes sur le travail des femmes. La définition même du concept de travail, la notion de stratégie et le thème du "temps " sont en effet au cour des analyses proposées par les différent(e)s auteur(e)s : autant d'objets cruciaux dans les recherches sur le travail féminin menées depuis le début des années 1980 .

Helena Hinata ouvre le recueil par une réflexion sur l'état des connaissances concernant la problématique et les théo- ries de la division sexuelle du travail dans les différentes disciplines des sciences sociales. Ce tour d'horizon, qui balaye les 25 dernières années, informe de façon synthétique sur les arguments qui ont conduit à questionner les concepts, les théories et les cloisonnements disciplinaires afin de déboucher sur une véritable problématique articulée des rapports sociaux de sexe. Ce texte d'ouverture offre une armature utile à l'ensemble de l'ouvrage. Marie-France Labrecque analyse ensuite la condition des femmes mexicaines et l'impact de la mondialisation des marchés dans la vie quotidienne des travailleuses de la transformation du maïs, ainsi que leur rôle dans la résistance zapatiste. Karen Messing et coll., dans une étude minutieuse des tâches accomplies par les enseignantes, nous montrent les stratégies de résistance utilisées par celles-ci pour faire face au manque de qualité du matériel didactique et garder le contrōle de la situation en devenant "la reine de la salle de classe ". Romaine Malenfant, quant à elle, examine d'une manière critique la "résistance tranquille " des travailleuses enceintes au Québec qui demandent le retrait préventif lorsqu'elles sont exposées à des conditions de travail dangereuses. L'auteure nous montre combien le patronat est réticent à s'ouvrir à la loi et aux changements dans l'organisation du travail pour que ces femmes puissent être assurées d'avoir une maternité sans danger au travail. Christine Corbeil et Francine Descarries nous présentent les résultats d'une vaste enquête menée auprès de femmes montréalaises et proposent un ensemble de réflexions sur la façon dont les mères travailleuses concilient travail salarié, travail domestique et exigences familiales. On peut ainsi voir se déployer une diversité de stratégies face aux contraintes du temps, au partage des tâches domestiques et au travail en général. Céline Séguin présente un portrait de mères seules montréalaises 
dans leur rapport au travail, qu'elle compare avec celui de mères travailleuses en couple : le défi de la gestion du temps est là aussi crucial. Angelo Soares expose la façon dont les caissières des supermarchés brésiliens et québécois font face à une organisation du travail "sans âme ", la solidarité qui existe entre elles et leurs différentes stratégies pour faire face à des clients violents, au harcèlement sexuel et leur lutte pour conserver le peu de contrôle qu'elles ont sur leur travail. Ana Maria Seifert et coll., recourant à une approche ergonomique pour analyser le travail des caissières de banque à Montréal, font ressortir les exigences au plan physique, mental et émotif de ce travail. Elles s'attachent également aux conséquences des vols de banque en termes de stratégies de support social développées entre les travailleuses suite à un vol ou une agression, et dans la construction du savoir nécessaire à l'accomplissement du travail. Johanne Prévost et Karen Messing, utilisant elles aussi une approche ergonomique, analysent le service 24 heures d'assistance téléphonique à la clientèle d'une entreprise canadienne des communications. Ce travail comporte des caractéristiques d'irrégularité et d'étalement qui représente un grand défi à la programmation d'activités régulières horstravail. Les résultats révèlent un ensemble de stratégies hors-travail dont l'enjeu est la possibilité de rester au travail. Et pour clôturer, Maria de Koninck propose sa lecture personnelle des textes et nous amène à nous questionner sur le sens du travail des femmes, pour les femmes.

L'ensemble de ces textes nous fournit ainsi un panorama très diversifié de ce qu'on regroupe sous un même vocable: stratégies de résistance. Il ressort de ces analyses, à mon sens, une sorte de continuum où la notion de résistance s'apparente d'un côté à la simple stratégie de survie et de l'autre, à une véritable "lutte silencieuse " contre les pressions des rouages de l'organisation. Vers le centre, on aurait les stratégies que l'on pourrait qualifier " d'aménagement " (les stratégies de conciliation famille-travail se trouveraient placées à cet endroit). Autant de formes de "résistance " vécues successivement ou simultanément par les travailleuses. Cet aspect polysémique de la notion de "stratégie de résistance" dans le contexte du travail des femmes m'apparait l'apport le plus substantiel de ce recueil bien construit et riche en données originales.

MARIANNE KEMPENEERS

Département de sociologie Université de Montréal

\section{Réduction du temps de travail : mode d'emploi}

par Jean-Pierre MONGRAND, Paris : Éditions d'Organisation, 1998, 342 p., ISBN

2-7081-2192-8.

S'il est un sujet qui a suscité autant le débat public que les publications en France, au cours des années 90, c'est bien la réduction du temps de travail. Le contexte difficile du marché du travail et l'opposition manifestée par plusieurs acteurs sociaux français à une stratégie de "flexibilisation" tous azimuts des règles de ce marché ont constitué le ferment de la recherche de stratégies autres pour contribuer à la réduction du chômage. C'est dans ce contexte qu'a ré-émergé le sujet de la réduction du temps de travail, considérée comme un mode particulier de régulation du marché du travail et posée comme une des formes de répartition des richesses. La première période de ce débat social, si l'on peut procéder à un tel découpage temporel, a donné lieu à de nombreuses publications à caractère tantôt plus théorique et tantôt plus idéologique. Dans la foulée des mesures législatives des dernières années, dont la loi incitative de juin 1996 (dite "Loi Robien"), puis celle plus directive du 13 juin 1998 (dite "Loi Aubry ") 